\title{
TEMPERATURE MEASUREMENTS BY OH LIF AND CHEMILUMINESCENCE KINETIC MODELING FOR ETHANOL FLAMES
}

Carla S. T. Marques*, Leila R. dos Santos, Maria E. Sbampato, Luiz G. Barreta e Alberto M. dos Santos

Divisão de Aerotermodinâmica e Hipersônica, Instituto de Estudos Avançados, Departamento de Ciência e Tecnologia Aeroespacial, Rod. dos Tamoios, km 5,5, 12228-001 São José dos Campos - SP, Brasil

Recebido em 21/10/08; aceito em 4/5/09; publicado na web em 1/10/09

\begin{abstract}
TEMPERATURE MEASUREMENTS BY OH LIF AND CHEMILUMINESCENCE KINETIC MODELING FOR ETHANOL FLAMES. OH LIF-thermometry was applied to premixed ethanol flames at atmospheric pressure in a burner for three flame conditions. Flame temperatures were simulated from energy equation with PREMIX code of CHEMKIN software package for comparison. A kinetic modeling based on a model validated through chemiluminescence measurements and on a set of reactions for nitrogen chemistry was evaluated. Marinov's mechanism was also tested. Sensitivity analysis was performed for fuel-rich flame condition with $\Phi=1.34$. Simulated temperatures from both reaction mechanisms evaluated were higher than experimental values. However, the proposed kinetic modeling resulted in temperature profiles qualitatively very close to the experimental.
\end{abstract}

Keywords: LIF-thermometry; chemiluminescence modeling; ethanol flames.

\section{INTRODUCTION}

The interest in ethanol has increased significantly in recent years because of environmental and health concerns. Ethanol is a renewable fuel and could be applied as a fuel extender, an oxygenate additive, an octane number enhancer and as a pure fuel. ${ }^{1,2}$

In Brazil, ethanol is extensively used such as gasoline additive and commonly direct as an automotive fuel in a large part of the national fleet. ${ }^{3,4}$ Nowadays, the use of ethanol has been increasing due to the "Flex fuel motors", in which the automotives vehicles can run on gasoline or ethanol fuels in any fraction. Recently, ethanol has also been applied as a pure fuel in small aircraft for aerial spraying in agriculture..$^{5-7}$

The increase of ethanol use, as the examples above, has stimulated several studies on ethanol oxidation through different techniques, wherein the main aims are the combustion evaluation and the pollutant formation. ${ }^{8-21}$ From these works, detailed reaction mechanisms have been proposed for ethanol combustion.

Temperature is one of the most important features for both combustion performance optimization (since it governs the chemical reaction rates) and pollutant formation processes. LIF-thermometry has been broadly employed to combustion research for accurate temperature determination..$^{22-25}$ The laser diagnostic ensures high spatial resolution without gas-dynamic disturbances into the flame. Furthermore, temperature is also a significant parameter to build reaction mechanisms to combustion modeling.

Combustion reactions could also be followed through chemiluminescence measurements. The chemiluminescent radicals are excellent probes of the combustion processes, in spite of their lower concentrations, because they are intermediate species with short lifetimes and consequently good markers of the reaction zones, in which the reagents are mostly consumed. Hence, their chemiluminescence measurements are suitable for following the combustion chemistry and other combustion features. One of them is the heat release rate and the chemiluminescent emissions have been usually used to provide qualitative information about it. ${ }^{26-34}$ Kinetic modeling is a diagnostic tool that could help in the interpretation of the chemiluminescence

*e-mail: carlatm@ieav.cta.br signals to achieve qualitative and quantitative information on parameters as combustion chemistry and heat release rates.

In this work, OH-LIF temperature measurements were carried out for different premixed ethanol flames at atmospheric pressure in a burner. Ethanol flames at stoichiometric and fuel-rich conditions were investigated. These ethanol burning processes were simulated with PREMIX code of CHEMKIN IV software package in order to obtain the temperature profiles, which were compared to the experimental ones. A reaction mechanism based on a model validated by chemiluminescence data ${ }^{35}$ and on a set of reactions for nitrogen chemistry ${ }^{36}$ was tested to reproduce the temperature profiles of premixed ethanol flames in a burner. Marinov's mechanism ${ }^{1}$ was also tested for evaluation purposes. Sensitivity analysis was performed to identify the main reactions that describe the temperature profiles and to help in the kinetic model optimization.

\section{EXPERIMENTAL}

\section{Burner configuration}

Ethanol flames were produced with a burner, in which the ethanol vapor/ $\mathrm{O}_{2} / \mathrm{N}_{2}$ mixture flows from a $4.5 \mathrm{~mm}$ diameter stainless-steel tube, where mixing occurs. Ethanol vapor flows through an orifice with $0.8 \mathrm{~mm}$ diameter inflowing in the burner tube with an angle of $52^{\circ}$. It begins mixing with $\mathrm{O}_{2} / \mathrm{N}_{2}$ reagents at $29.5 \mathrm{~mm}$ of the burner tube length. The burner tube lengths $69.5 \mathrm{~mm}$ long and the burner has an external diameter of $43.0 \mathrm{~mm}$. From a heating system, ethanol was vaporized and the burner was kept warmed up at $120{ }^{\circ} \mathrm{C}$ to prevent condensation on the walls, where the combustible mixture flows.

\section{Flame conditions}

Three premixed ethanol vapor $/ \mathrm{O}_{2} / \mathrm{N}_{2}$ flames at atmospheric pressure in a burner were investigated. One of them at stoichiometric condition and the other two at fuel-rich conditions. Table 1 shows the premixed ethanol flame conditions studied.

The combustible mixtures were prepared and flowed into the burner through rotameters. The flowmeters were previously calibrated for each reagent fluid at work pressure and temperature with a flow 
calibration apparatus (Hastings, model HBM-1A). The calibration measurements of the flowmeters showed ca. $3-4 \%$ errors.

Table 1. Ethanol vapor $/ \mathrm{O}_{2} / \mathrm{N}_{2}$ flame conditions

\begin{tabular}{lcccc}
\hline$\dot{V}_{E I O H} / \mathrm{cm}^{3} \mathrm{~min}^{-1}$ & $\dot{V}_{O 2} / \mathrm{cm}^{3} \mathrm{~min}^{-1}$ & $\dot{V}_{N 2} / \mathrm{cm}^{3} \mathrm{~min}^{-1}$ & $\% \mathrm{~N}_{2}(\mathrm{v} / \mathrm{v})$ & $\Phi^{\#}$ \\
\hline 2.57 & 3116 & 5621 & 64.3 & 1.02 \\
2.57 & 2374 & 4432 & 65.1 & 1.34 \\
2.57 & 1911 & 3638 & 65.5 & 1.66 \\
\hline$\#=\frac{\dot{n}_{E t O H} / \dot{n}_{O 2}}{S . R .}$, where S. R. is the stoichiometric ratio to the \\
$\mathrm{C}_{2} \mathrm{H}_{5} \mathrm{OH}+3 \mathrm{O}_{2}=2 \mathrm{CO}_{2}+3 \mathrm{H}_{2} \mathrm{O}$ reaction.
\end{tabular}

Ethanol fuel was vaporized for the flowmeter calibration and combustible mixture burning through a heating system.

\section{LIF measurements}

The experimental setup for OH-LIF measurements is similar as those used in Santos. ${ }^{37}$ For temperature measurements, the excitation spectra were acquired at different flame regions and the Boltzmann's method was applied to establish the temperature values. ${ }^{37}$

A $30 \mathrm{~Hz}$ pulsed Nd:YAG laser (Continuum, model Powerlite PL7030) at $532 \mathrm{~nm}$ was used to pump a frequency-doubled dye laser (Continuum, model Jaguar JAG60MA) with rhodamine 6G. The laser beam tuned in the $\mathrm{A}^{2} \Sigma^{+}-\mathrm{X}^{2} \Pi(1,0)$ band around $280 \mathrm{~nm}$ was enough attenuated by adequate filters and was focused with an UV-lens $(f=300$ $\mathrm{mm}, f / 5.9$ ) into the flame for linear excitation of the $\mathrm{OH}$ molecules. The energy of beam laser was monitored by an energy meter (Ophir, model PE10). It was in the range of 50-90 $\mu \mathrm{J} /$ pulse. The $\mathrm{OH}$ laser-induced fluorescence was collected at $90^{\circ}$ and focused with an $f=250 \mathrm{~mm}, f$ 19.8 UV-lens on a 1/8 m monochromator (Spex, model H10). The $\mathrm{OH}$ light emission was collected spectrally integrated in the $\mathrm{A}^{2} \Sigma^{+}-\mathrm{X}^{2} \Pi(0,0)$ band, centered at $318 \mathrm{~nm}$ with bandwidth of $32 \mathrm{~nm}$ and was detected by a photomultiplier (Hamamatsu, R928). The optical configuration of the detection system was properly adjusted to avoid spontaneous emission background of the flames. The laser signal detected by a fast photodiode was used to trigger a boxcar integrator (Stanford Research Systems, SR200) for analyzing the photomultiplier signals. The signals were sampled by a SR250 acquisition boxcar module setting a gatewidth of $10 \mathrm{~ns}$. At this integration window, the maximum of the fluorescence was centered after $40 \mathrm{~ns}$. The signals were acquired in the boxcar integrator with a $50 \Omega$ terminator.

The scanning of the $\mathrm{OH}$ excitation spectra was carried out in the range of 278.5-281.0 nm with stepwidth rate of $0.005 \mathrm{~nm} / \mathrm{s}$. This spectral region corresponds to the rotational lines $S_{21}(1)$ to $S_{21}(13)$ in the $A^{2} \Sigma^{+}-X^{2} \Pi(1,0)$ vibronic transition. Each point of the excitation spectra is an average value of the induced fluorescence intensity for 30 laser shots. The temperature for each axial position in the flame was determined by the relationship between Boltzmann distribution and temperature for the rotational lines above. ${ }^{37} \mathrm{OH}$ excitation spectra were obtained for three premixed ethanol flames to establish the temperature axial profiles.

\section{SIMULATION}

The chemical kinetic model for ethanol combustion was simulated through PREMIX code of CHEMKIN IV software package ${ }^{38,39}$ to predict temperature profiles in premixed ethanol flames. Premixed laminar burner-stabilized flame was considered as reactor model. PREMIX program accounts for finite-rate chemical kinetics and mixture-averaged or multi-component molecular transport. It solves the set of governing differential equations that describes the flame dynamics using implicit finite difference methods, as well as, a combination of time-dependent and steady-state methods. ${ }^{38,39}$

The numerical solution procedure begins by making finite difference approximations to reduce the boundary value problem to a system of algebraic equations, from an initial estimate provided by the user. The starting estimate is based on the occurrence of a reaction zone that separates the unburned reagent mixture from the burned combustion products. At the end of the reaction zone, the major portion of the reagents was converted to the products. In our simulations, the CHEMKIN calculated an equilibrium composition to be used as the combination products estimate. Therefore, the first solution uses the initial temperature profile as a fixed-temperature constraint and only solves for the species composition and the velocity profile. The second solution solves the fully coupled reacting-flow and energy equations for premixed flames. ${ }^{38,39}$

The PREMIX program attempts to solve the system of algebraic equations by the damped modified Newton algorithm in TWOPNT program. ${ }^{40}$ However, if the Newton algorithm fails to converge, the solution estimate is conditioned by integration in time. In this case, TWOPNT solves the transient equations for a given number of time steps, and then returns to the Newton method using the result of the time-stepping as the new initial iterate. The program solves this system of ordinary differential equations using the backward-Euler method. In this method, the time derivatives are approximated by first-order, backwards finite differences. To solve the system of equations for each time step, the same Newton method employed in the steady-state problem is used..$^{38,39}$

Here, heat losses were not considered to simulate the temperature profiles; although in many flames there can be significant heat losses to the external environment. However, they are of unknown or questionable origin and thus are difficult to model. ${ }^{38}$

The first-order sensitivity coefficients of gas temperature were also calculated by PREMIX program for fuel-rich premixed ethanol flame with $\Phi=1.34$. Sensitivity analysis is the calculation of the change rates in the output variables of a model, which result from small perturbations in the model parameters. ${ }^{39,41}$ In PREMIX code, the sensitivity coefficients are calculated from Jacobian matrix, $\mathrm{J}=\partial \mathrm{F} / \partial \phi$, that is the iteration matrix of the original system, where $F$ is the residual vector and $\phi$ is the vector of temperature and mass fractions. The first-order sensitivity coefficient matrix is defined as $S_{1, i}=\partial \phi / \partial \alpha_{i}$, where 1 and $i$ are, respectively, dependent variables and reactions and $\alpha_{i}$ is the parameter vector that represents the pre-exponential A-factors in the Arrhenius reaction-rate expressions. The sensitivity coefficient matrix contains quantitative information on how each reaction rate constant affects the temperature and/or species fractions. For more useful information, the normalized sensitivity coefficients are calculated as logarithmic derivatives, i.e., for temperature they are $\left.\partial \ln T / \partial \ln \alpha_{i}=\left(\alpha_{i} / \mathrm{T}\right)\left(\partial \mathrm{T} / \partial \alpha_{\mathrm{i}}\right)\right)^{38,39}$ From sensitivity analysis, it was possible to evaluate the set of reactions, which strongly contribute to the temperature profiles.

\section{REACTION MECHANISM}

The reaction mechanism for ethanol combustion was developed to reproduce experimental temperatures, NO concentrations and chemiluminescent emission profiles in premixed flames. It was built from two models: one for ethanol combustion in a closed chamber, which was validated through chemiluminescent emissions' data $^{35}$ and other related to nitrogen chemistry for reburning process to NO reduction in a furnace. ${ }^{36}$

To these set of reactions, 20 ones for $\mathrm{OH}^{*}$ and $\mathrm{CH}^{*}$ non-radiative decays were inserted ${ }^{42-44}$ although they have been discarded previously. ${ }^{35,42}$ Two new reactions proposed for $\mathrm{OH}^{*}$ formation in the recent works of Smith et al. $^{45}$ and Harber and Vandsburger ${ }^{46}$ was also considered. 
Thermodynamic data for electronically excited species were calculated through FITDAT program of CHEMKIN software package ${ }^{38}$ from thermodynamic coefficients of the species in their respective ground states, adding the photon energy, hv, for each radiative transition to the enthalpy of each ground state at $298 \mathrm{~K}$. For $\mathrm{CH}_{2} \mathrm{CN}$ radical the thermodynamic coefficients were also calculated ${ }^{36}$ using FITDAT, but the molecular vibrational frequencies were the inputs for fitting procedure. The thermodynamic coefficients calculated by FITDAT were fitted to the $300-5000 \mathrm{~K}$ temperature range with equality constraint of $1000 \mathrm{~K}$ as those in the reaction mechanism.

The initial mechanism consists of 63 species and 325 elementary reactions. The reaction set and kinetic parameters are not presented here, because they are only recommended for qualitative simulation purposes. Its optimization, reduction and validation will be evaluated later through experimental NO concentration and chemiluminescent emission profiles in premixed ethanol flames, as will be explained further on. At first, the ability of this initial reaction mechanism to reproduce experimental temperature profiles for premixed ethanol flames was tested in this work.

\section{RESULTS AND DISCUSSION}

The experimental temperatures obtained by OH-LIF spectroscopy resulted in accurate measurements, as it was supposed. The temperature deviation was estimated to be around $\pm 150 \mathrm{~K}( \pm 6 \%)$ for the largest error found in our measurements. The uncertainties in the temperature values are mainly due to the flame fluctuations (uncertainty of the flow rates, slight ethanol flow rate decrease during experiment, surrounding air diffusion, etc.)

Although ethanol flame with $\Phi=1.66$ consists of a very fuelrich flame condition, no significant background interferences on LIF signals would be observed for low-energy measurements, as reported by Reisel et al. for a very similar ethane flame condition. ${ }^{47}$ Furthermore, ethanol is an oxygenated fuel and there is still lower soot production from its oxidation.

Experimental and simulated temperature profiles for stoichiometric $(\Phi=1.02)$ and fuel-rich $(\Phi=1.34$ and $\Phi=1.66)$ premixed ethanol flames in a burner are presented in Figure 1.

The experimental profiles for the three flame conditions studied (Figure 1a) have the same behavior as those predicted by the theory of premixed laminar flame, in which the temperature increases in the pre-heating and reaction zones up to the final value, where the reagents were almost fully consumed. Thus, sigmoidal curves were well fitted to the experimental temperature values, as shown in Figure 1a.

From first-derivatives applied to the fitted sigmoidal profiles (Figure 1a), it was possible to establish approximately the end of the reaction zones for fuel-rich flame conditions: $20 \mathrm{~mm}$ in $\Phi=$ 1.34 and $45 \mathrm{~mm}$ in $\Phi=1.66$. As the temperature measurements have been acquired in much spaced flame regions, these values are only estimates. For stoichiometric ethanol flame, the end of the reaction zone is undetermined from sigmoidal profile; the first-derivative never goes to zero.

Simulated temperature profiles from two different kinetic modeling: EtOH-Chem-NO (this work) and Marinov's ${ }^{1}$ mechanism were obtained, as shown in Figures $1 \mathrm{~b}$ and $1 \mathrm{c}$. Both reaction mechanisms resulted in temperature values higher than experimental temperatures for all simulated flame conditions. They are also higher than adiabatic temperatures. The higher simulated temperatures are due to absence of heat loss in calculations or to the choice of the reaction sets, which are adding further energy to the system.

Table 2 displays experimental and simulated temperature values for premixed ethanol flames in a burner at different flame regions for better evaluation.
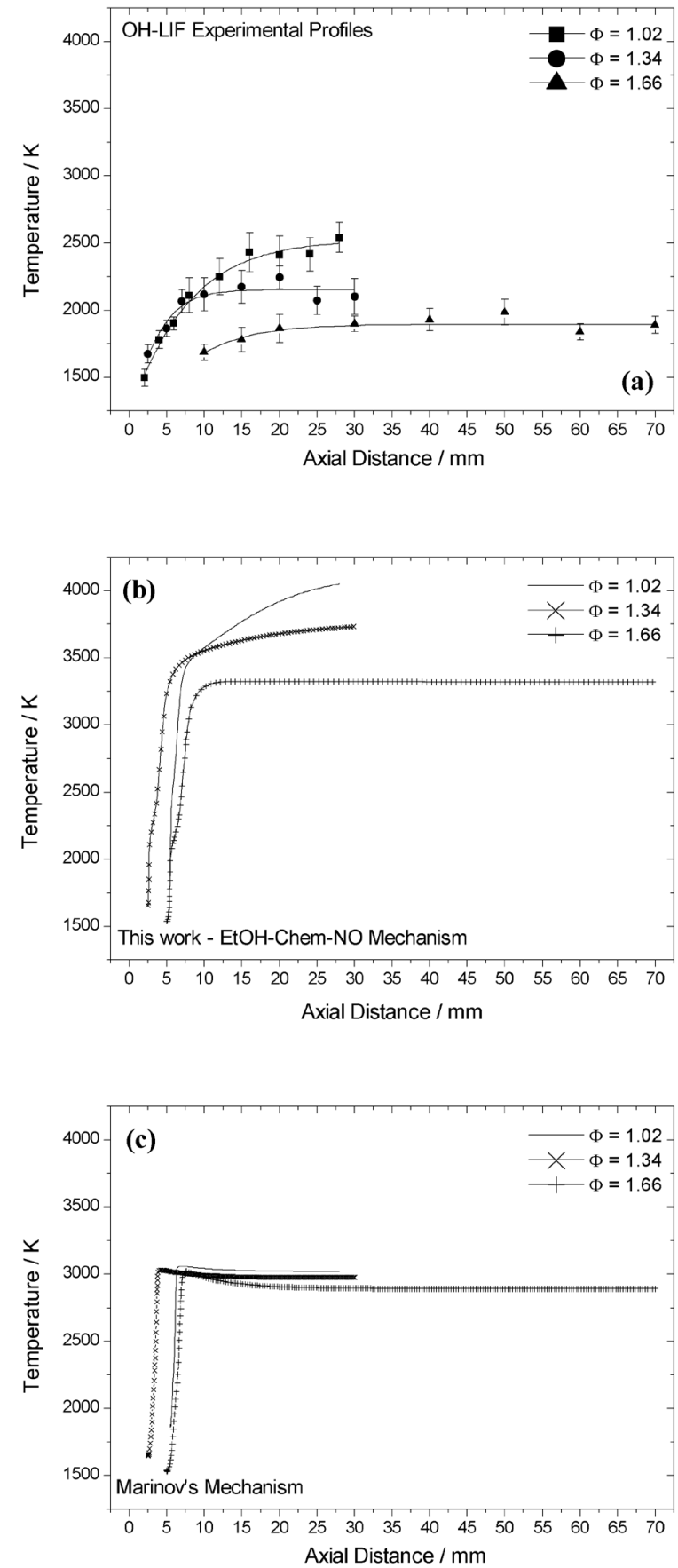

Figure 1. Temperature profiles for premixed ethanol flames in a burner: (a) experimental; (b) simulated by EtOH-Chem-NO mechanism (this work) and (c) simulated by Marinov's mechanism

At the end of the linear part of the temperature profiles, analogous temperature values were simulated by both reaction mechanisms (considering experimental deviation), but they have been very higher than those experimental. Otherwise, the Marinov's mechanism simulated lower values for maximum temperature.

Although the higher temperature values simulated by our reaction mechanism as final temperature, the behavior of the simulated profiles (Figure 1b) is very close to the experimental ones (Figure 1a). Only the simulated profile for premixed ethanol flame with $\Phi=$ 1.66 is not so similar to the experimental, due to the mechanism does not include reactions with higher $\mathrm{C}$-species that should represent the benzene species growth and a slower global chemical reaction rate. 
Table 2. Simulated and experimental temperatures for premixed ethanol flames in a burner

\begin{tabular}{|c|c|c|c|c|c|c|c|}
\hline \multirow{3}{*}{$\frac{\text { Ethanol Flame }}{\Phi}$} & \multicolumn{3}{|c|}{$\mathrm{T}_{\text {linear curve end }} / \mathrm{K}$} & \multicolumn{3}{|c|}{$\mathrm{T}_{\text {maximum }} / \mathrm{K}$} & \multirow{3}{*}{$\frac{\mathrm{T}_{\text {adiabatic }} / \mathrm{K}}{\text { Calculated by Gaseq* }}$} \\
\hline & \multicolumn{2}{|c|}{ Simulated } & \multirow{2}{*}{ Exp. } & \multicolumn{2}{|c|}{ Simulated } & \multirow{2}{*}{ Exp. } & \\
\hline & Marinov & This work & & Marinov & This work & & \\
\hline 1.02 & 3039 & 3228 & $2111 \pm 131$ & 3059 & 4049 & $2541 \pm 112$ & 2640 \\
\hline 1.34 & 3013 & 3134 & $2068 \pm 84$ & 3031 & 3732 & $2244 \pm 83$ & 2590 \\
\hline 1.66 & 2937 & 3046 & $1863 \pm 106$ & 3019 & 3320 & $1983 \pm 95$ & 2355 \\
\hline
\end{tabular}

* Gaseq (v. 0.79) is software that calculates the chemical equilibrium in perfect gases. The thermodynamic data from Marinov et al. ${ }^{48}$ for gas ethanol were employed in the calculations.

The initial reaction mechanism built is qualitatively better than Marinov's mechanism for temperature simulations, because it was based on an ethanol kinetic model validated through chemiluminescence measurements ${ }^{35}$ and they follow very well the heat release rates in combustion processes. This fact can be observed through sensitivity coefficients of gas temperature.

Figure 2 presents the first-order sensitivity coefficients of gas temperature for premixed ethanol flame with $\Phi=1.34$ at different simulated flame regions.

The set of reactions that describes the temperature profiles is that evolved in the chain reaction of ethanol combustion, including some chemiluminescence reactions ${ }^{35}$ as identified by the sensitivity analysis (Figure 2).

As it can be seen in Figure 2, the reactions with higher sensitivity coefficients of temperature are those found at simulated flame region around $4.3 \mathrm{~mm}$. These 24 reactions have strong influence on simulated temperature profiles and are practically accountable to describe them. Reactions: R7, R8, R13, R14, R37 and R43 are those with large contribution, since they have temperature sensitivity coefficients higher than -0.1 or +0.1 .

The first rising temperature up to ca. $2.6 \mathrm{~mm}$ is other simulated flame region, where some reactions with high sensitivity coefficients are recognized. Part of them is related to the ethanol molecule degradation and oxidation (R16, R23 and R24), as presented in Figure 2.

Only R16 has temperature sensitivity coefficient higher than +0.1 , which was reported as the major reaction for ethanol consumption by Norton and Dryer ${ }^{18,20}$ and Benvenutti et al. ${ }^{35}$

Furthermore, there are two chemiluminescence reactions (R287 and R305) that have significant contribution to the simulated temperature profiles with sensitivity coefficients next to +0.1 .

To better fit the simulated temperature profiles to the experimental data, the kinetic modeling should be optimized. The first trials designed to perform this were the changes in the rate constants for the reactions with higher temperature sensitivity coefficients within their error limits. PREMIX was run several times to implement these changes which could only reduce the increasing temperature up to about 400-500 K, but they have almost not effect on the final temperature. In spite of lower simulated temperatures, the behavior of the profiles was disturbed.

The reaction set of a kinetic model is strongly dependent on the combustion process studied, mainly due to reaction rates and pressure. The reaction mechanism built was based on reaction subsets ${ }^{35,36}$ for very different combustion processes than those studied here. Hence, several changes in the reaction set should be made to reach an optimized kinetic model that describes properly the combustion processes studied.

Therefore, to improve the kinetic modeling optimization, the profiles of combustion intermediate species ought to be taken into account, since they can describe, kinetically, better the combustion process than the temperature profiles. For future modeling reduction, optimization and validation, experimental NO concentration and chemiluminescent emission profiles in premixed ethanol flames must
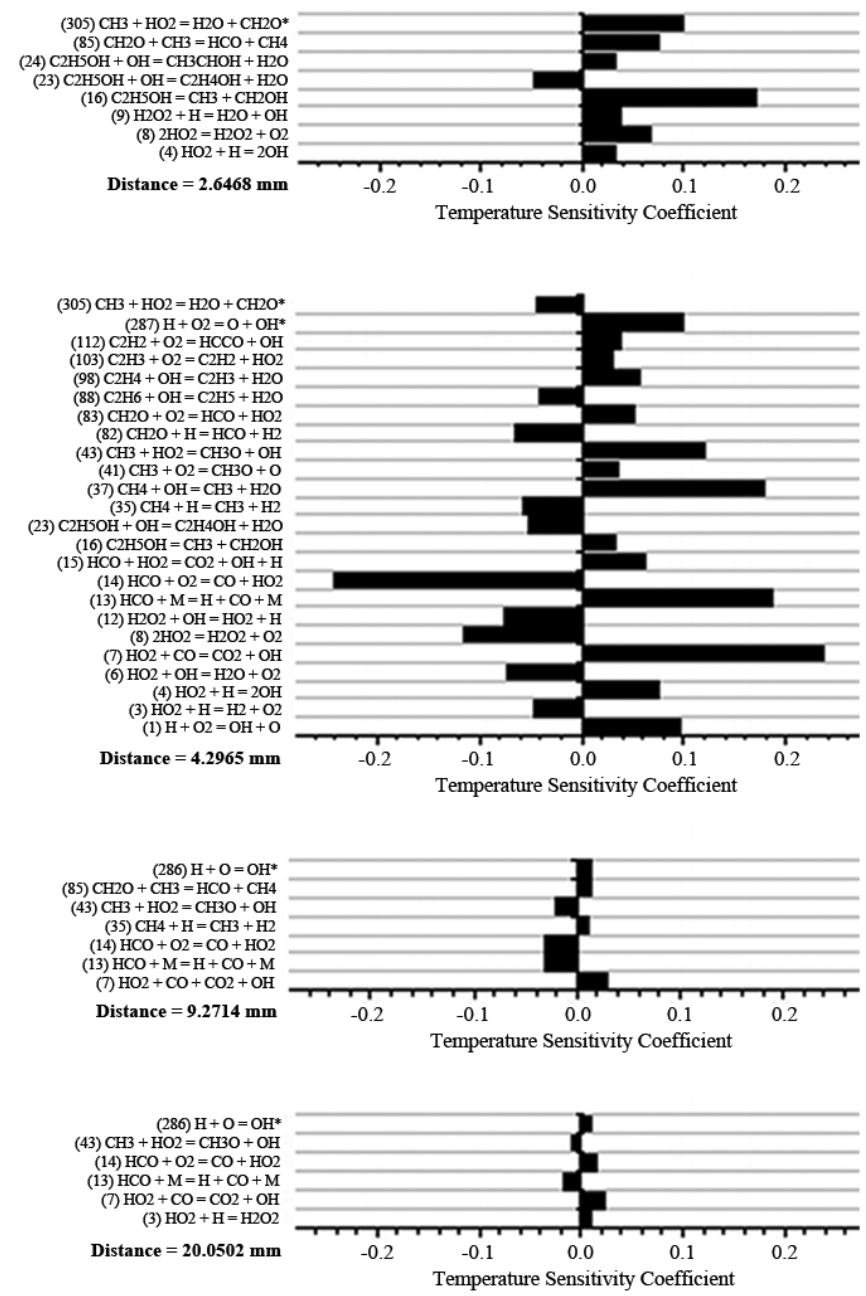

Figure 2. First-order sensitivity coefficients of gas temperature at different axial distances for premixed ethanol flame with $\Phi=1.34$

be considered. After these optimization and validation procedures simultaneously with temperature sensitivity analysis, it is supposed to achieve simulated temperature profiles with quantitatively enhanced agreement to the experimental ones for premixed ethanol flames.

\section{CONCLUSIONS}

The experimental temperature profiles obtained by OH LIFthermometry for the stoichiometric and fuel-rich premixed ethanol flames follow those predicted by the theory of premixed laminar flames and sigmoidal curves were well fitted to them.

Both reaction mechanisms simulated to reproduce temperature profiles for the ethanol flames studied through PREMIX code of CHE- 
MKIN program resulted in temperature values very higher than experimental temperatures measured by OH-LIF spectroscopy. Although the poor quantitative agreement, the kinetic modeling proposed produced simulated profiles qualitatively very close to the experimental ones and better than those obtained by Marinov's mechanism.

Sensitivity analysis indicated that the temperature profiles are described through 24 reactions, the largest part of them evolved in ethanol combustion and some in the chemiluminescence formation. The qualitatively well-shaped profiles simulated by our reaction mechanism are due to the strong relationship between chemiluminescence measurements and heat release rates, as supposed.

Qualitative information by simulations is useful and important to predict previously optimized combustion parameters in practical devices as, for example, engine performance as function of operation conditions. However, quantitative information is essential to specify this optimal combustion condition in practical systems.

To improve the quantitative predictions of the kinetic modeling, optimization and validation procedures through experimental $\mathrm{NO}$ concentrations and chemiluminescent emissions simultaneously with temperature sensitivity analysis are required.

\section{SUPPLEMENTARY MATERIAL}

In addition, a photograph of the burner and its heating system used to generate the premixed ethanol flames (Figure 1S), a scheme of experimental setup for temperature measurements by OH-LIF (Figure $2 \mathrm{~S}$ ) and a plot of simulated temperature profiles after changes in the kinetic modeling (Figure 3S) are found as supplementary material. They are available free of charge at http://www.quimicanova.sbq. org.br, in PDF file.

\section{ACKNOWLEDGMENTS}

The authors are thankful to LAS/INPE (Associated Laboratory of Sensors and Materials of the National Institute of Spatial Research) and to Dr. E. Corat for his assistance. This work was supported by FAPESP (The State of São Paulo Research Foundation) through project 2005/00505-9.

\section{REFERENCES}

1. Marinov, N. M.; Int. J. Chem. Kin. 1999, 31, 183.

2. Kasper, T. S.; Oßwald, P. O.; Kamphus, M.; Kohse-Höinghaus, K.; Combust. Flame 2007, 150, 220.

3. Pimentel, A. S.; Arbilla, G.; Quim. Nova 1997, 20, 252.

4. Pereira, P. A. P.; Santos, L. M.; Sousa, E. T.; Andrade, J. A.; J. Braz. Chem. Soc. 2004, 15, 646.

5. http://www.defesanet.com.br/embraer/neiva/, accessed in September 2009.

6. http://www.ethanolproducer.com/article.jsp?article_id=607, accessed in September 2009.

7. http://www.embraer.com.br/institucional/download/2_083-Prd-VPIEthanol_Ipanema_Certification-I-04.pdf, accessed in September 2009.

8. Boyarshinov, B. F.; Titkov, V. I.; Fedorov, S. Yu.; Combust., Expl. Shock Waves 2005, 41, 379.

9. Varde, K.; Jones, A.; Knutsen, A.; Mertz, D.; Yu, P.; Proc. IMechE - Part D: J. Automobile Eng. 2007, 221, 933.

10. Alzueta, M. U.; Hernández, J. M.; Energy Fuels 2002, 16, 166.

11. Taylor, P. H.; Cheng, L.; Dellinger, B.; Combust. Flame 1998, 115, 561.

12. Taylor, P. H.; Cheng, L.; Dellinger, B.; SAE Tech. Papers 1996, No. 961088.

13. Flowers, D. L; Aceves, S. M.; Frias, J. M.;. SAE Tech. Papers 2007, No. 2007-01-1867.
14. Xingcai, L.; Zhen, H.; Wugao; Degang, L.; Combust. Sci. Tech. 2004, 176,1309

15. Yozgatligil, A.; Park, S. H.; Choi, M. Y.; Kazakov, A.; Dryer, F. L.; Combust. Sci. Tech. 2004, 176, 1985.

16. Dunphy, M. P.; Simmie, J. M.; J. Chem. Soc. Faraday Trans. 1991, 87, 1691.

17. Tanoff, M. A.; Schear, D. M.; Olsson, J. O.; Anderson, L. A.; Bull. Soc. Chim. Belg. 1992, 101, 839

18. Norton, N. S.; Dryer, F. L.; Int. J. Chem. Kinet. 1992, 24, 319.

19. Dunphy, M. P.; Patterson, P. M.; Simmie, J. M.; J. Chem. Soc. Faraday Trans. 1991, 87, 2549.

20. Norton, N. S.; Dryer, F. L.; Proc. Combust. Inst. 1990, 23, 179.

21. Dagaut, P.; Boettner, J. C.; Cathonet, M.; J. Chim. Phys. 1992, 89, 867.

22. Boyarshinov, B. F.; Fedorov, S. Y.; Combust., Expl. Shock Waves 2004, 40, 511.

23. Mercier, X.; Pillier, L.; Bakali, A. E.; Carlier, M.; Pauwels, J. F.; Desgroux, P.; Faraday Discuss. 2001, 119, 305.

24. Palma, P. C.; Danehy, P. M.; Houwing, A. F. P.; AIAA J. 2003, 41, 1722.

25. Meier, U. E.; Wolff-Gaßmann, D.; Stricker, W.; Aerosp. Sci. Technol. 2000, 4, 403.

26. Yip, B.; Samaniego, J. M.; Combust. Sci. Tech. 1992, 84, 81.

27. Xiao, X.; Choi, C. H.; Puri, I. K.; Combust. Flame 2000, 120, 318

28. Hardalupas, Y.; Orain, M.; Combust. Flame 2004, 139, 188.

29. Buechner, H., Hirsch, C.; Leuckel, W.; Combust. Sci. Tech. 1993, 94, 219.

30. Keller, J. O.; Saito, K.; Combust. Sci. Tech. 1987, 53, 137.

31. Poinsot, T. J.; Trouvé, A. C.; Veynante, D. P.; Candel, S. M.; Esposito, E. J.; J. Fluid Mech. 1987, 177, 265.

32. Bloxsidge, G. J.; Dowling, A. P.; Langhorne, P. J.; J. Fluid Mech. 1988, $193,445$.

33. Keller, J. O.; Hongo, I.; Combust. Sci. Tech. 1990, 80, 219

34. Yoshida, A.; Narisawa, M.; Tsuji, H.; Hirose, T.; JSME Int. J. B. 1995, $38,222$.

35. Benvenutti, L. H.; Marques, C. S. T.; Bertran, C. A.; Combust. Sci. Tech. $\mathbf{2 0 0 5}, 177,1$.

36. Bertran, C. A.; Marques, C. S. T.; Vergnhanini Filho, R.; Fuel 2004, 83, 109.

37. Santos, L. R.;Tese de Doutorado, Universidade de São Paulo, Brasil, 2005.

38. Kee, R. J.; Rupley, F. M.; Miller, J. A.; Coltrin, M. E.; Grcar, J. F.; Meeks, E.; Moffat, H. K.; Lutz, A. E.; Dixon-Lewis, G.; Smooke, M. D.; Warnatz, J.; Evans, G. H.; Larson, R. S.; Mitchell, R. E.; Petzold, L. R.; Reynolds, W. C.; Caracotsios, M.; Stewart, W. E.; Glarborg, P.; Wang, C.; McLellan, C. L.; Adigun, O.; Houf, W. G.; Chou, C. P.; Miller, S. F.; Ho, P.; Young, P. D.; Young, J.; Hodgson, D. W.; Petrova, M. V.; Puduppakkam, K. V.; CHEMKIN Release 4.1.1, San Diego, CA, U.S.A., 2007.

39. Kee, R. J.; Grcar, J. F.; Smooke, M. D.; Miller, J. A.; Sandia National Laboratories, Report SAND85-8240, 1985.

40. Grcar, J. F.; Sandia National Laboratories, Report SAND91-8230, 1996.

41. Petzold, L.; Cao, Y.; Li, S.; Serban, R.; Lawrence Livermore National Laboratory, Report UCRL-PROC-214507, 2005.

42. Marques, C. S. T.; Benvenutti, L. H.; Bertran, C. A.; J. Braz. Chem. Soc. 2006, 17, 302 .

43. Hall, J. M.; Rickard, M. J. A.; Petersen, E. L.; Combust. Sci. Tech. 2005, $177,455$.

44. Smith, G. P.; Luque, J.; Park, C.; Jeffries, J. B.; Crosley, D. R.; Combust. Flame 2003, 131, 59

45. Smith, G. P.; Park, C.; Luque, J.; Combust. Flame 2005, 140, 385.

46. Harber, L. C.; Vandsburger, U.; Combust. Sci. Tech. 2003, 175, 1859.

47. Reisel, J. R.; Carter C. D.; Laurendeau, N. M.; Drake, M. C.; Combust. Sci. Tech. 1993, 91, 271.

48. Marinov, N. M.; Pitz, W.J.; Westbrook, C.K.; Hori, M.; Matsunaga, N.; Proc. Combust. Instit. 1998, 27, 389. 


\section{TEMPERATURE MEASUREMENTS BY OH LIF AND CHEMILUMINESCENCE KINETIC MODELING FOR} ETHANOL FLAMES

Carla S. T. Marques*, Leila R. dos Santos, Maria E. Sbampato, Luiz G. Barreta e Alberto M. dos Santos

Divisão de Aerotermodinâmica e Hipersônica, Instituto de Estudos Avançados, Departamento de Ciência e Tecnologia Aeroespacial, Rod. dos Tamoios, km 5.5, 12228-001 São José dos Campos - SP, Brasil

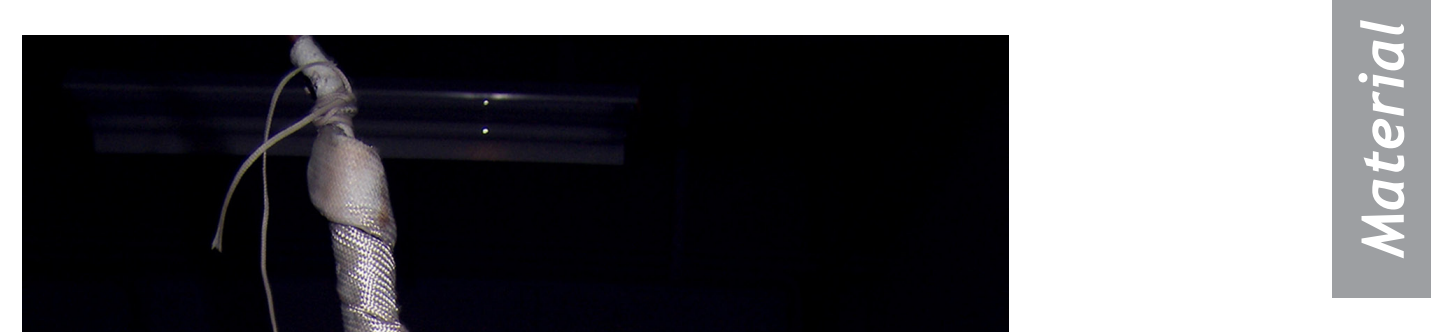

Figure 1S. Photograph of the burner and its heating system to generate the ethanol vapor $/ \mathrm{O}_{2} / \mathrm{N}_{2}$ flames 


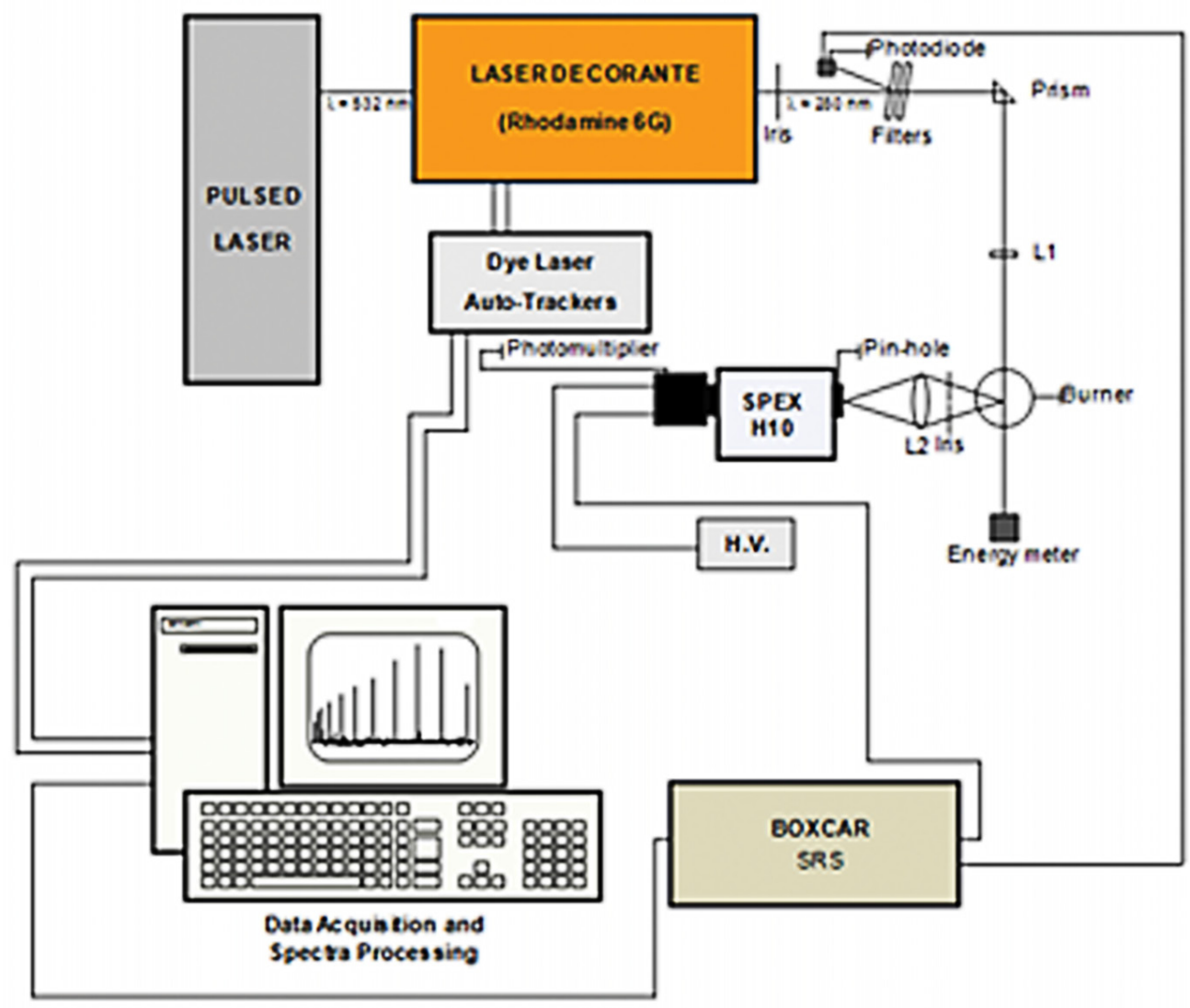

Figure 2S. OH LIF schematic diagram for temperature measurements

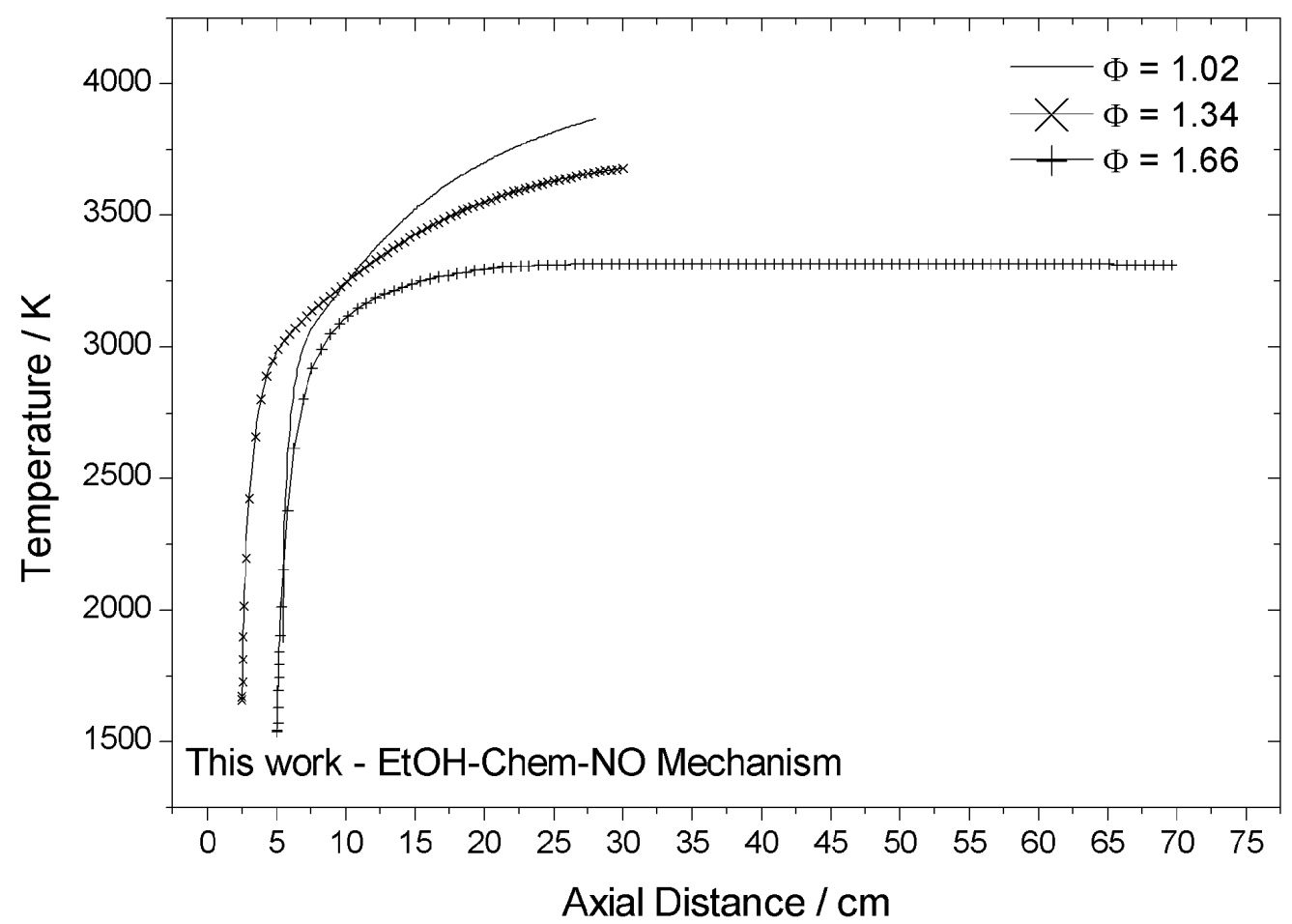

Figure 3S. Simulated temperature profiles after changes in the reaction mechanism. Rate constants of reactions (3), (4), (7), (9), (12), (14), (24), (43), (82), (88) e (98) were modified within their error limits 